\title{
1 Olfactory detection and discrimination in domestic dogs (Canis lupus familiaris)
}

3 Elodie Ferrando ${ }^{1,2,3}$ and Christoph D. Dahl ${ }^{1,4,5, *}$

4

5

${ }_{6}{ }^{1}$ Institute of Biology, University of Neuchâtel, Neuchâtel, Switzerland

$7{ }^{2}$ MTA-ELTE 'Lendület' Neuroethology of Communication Research Group - Eötvös

8 Loránd University, H-1117 Budapest, Pázmány Péter sétány 1/C, Hungary

$9^{3}$ Department of Ethology, Doctoral School of Biology, Institute of Biology, ELTE Eötvös

10 Loránd University, H-1117 Budapest, Pázmány Péter sétány 1/C, Hungary

${ }_{11}^{4}$ Graduate Institute of Mind, Brain and Consciousness, Taipei Medical University, Taipei,

12 Taiwan

${ }_{13}^{5}$ Brain and Consciousness Research Center, Taipei Medical University Shuang-Ho Hospital,

14 New Taipei City, Taiwan

15

16

${ }_{17}^{*}$ * corresponding author: Christoph D. Dahl <christoph.d.dahl@gmail.com> 


\section{${ }_{18}$ Abstract}

зз Keywords

The extraordinary olfactory capabilities in detection and rescue dogs are well-known. However, the olfactory performance varies by breed and search environment (Jezierski et al., 2014), as well as by the quantity of training (Horowitz et al., 2013). While detection of an olfactory cue inherently demands a judgment regarding the presence or absence of a cue at a given location, olfactory discrimination requires an assessment of quantity, a task demanding more attention and, hence, decreasing reliability as an informational source (Horowitz et al., 2013). This study aims at gaining more clarity on detection and discrimination of olfactory cues in untrained dogs and in a variety of dog breeds. Using a two-alternative forced choice (2AFC) paradigm, we assessed olfactory detection scores by presenting a varied quantity of food reward under one or the other hidden cup, and discrimination scores by presenting two varied quantities of food reward under both hidden cups. We found relatively reliable detection performances across all breeds and limited discrimination abilities, modulated by breed. We discuss our findings in relation to the cognitive demands imposed by the tasks and the cephalic index of the dog breeds.

Olfaction, dog, Canis lupus familiaris, quantity discrimination, detection 


\section{Introduction}

The dog's high sensitivity of the olfactory system might subserve a particular fitness-enhancing trait present in the evolutionary ancestor of today's canids, i.e. the detection of a prey in relative close proximity (Horowitz et al., 2013) or the inference about the location of a distal prey via airborne olfactory particles (Hepper and Wells, 2005). Apart from that, domestic dogs use olfaction in social contexts, such as social interactions (Bräuer and Blasi, 2021), identity recognition (Cafazzo et al., 2012; Lisberg and Snowdon, 2009), intrasexual competition for mates, territorial defence (Bidder et al., 2020) and parental care (Foyer et al., 2016; Lévy et al., 2004). In general, the olfactory bulb and its projection structures, including the olfactory tract and striae, are larger in dogs than in humans in absolute terms and relative to brain size (Kavoi and Jameela, 2011), suggesting higher olfactory functionality (Haehner et al., 2008). In numbers, olfactory receptors are 30 times more in $\operatorname{dogs}(\approx 200$ million) compared to humans $(\approx 6$ million) (Horowitz, $2014 ;$ Lindsay, 2013). In particular, however, the actual size of the olfactory epithelium varies largely across breeds (Quignon et al., 2003), paralled by inter-breed differences in behavioural outcome: Dog breeds selected for olfactory tasks (scenting breeds) and wolves performed better in detecting one of four pots baited with a food reward than short-nosed and non-scent breeds (Polgár et al., 2016).

Despite a general consensus on the importance of olfaction in dog cognition, relatively little investigation has been conducted regarding some of the most fundamental perceptual abilities, such as discrimination of two scents, one of them stronger than the other (Becker et al., 1962; Cablk et al., 2008). Research programmes focused predominantly on visuo-cognitive questions, such as visual human-dog communication (Kerepesi et al., 2005; Horowitz, 2021) and interpretation of pointing gestures (Soproni et al., 2002). Likewise, most insights into quantity discrimination in dogs rose from visual numerical discrimination (Prato-Previde et al., 2008; Petrazzini and Wynne, 2016, 2017). These studies showed that dogs, including puppies (Petrazzini et al., 2020), tend to select significantly more often the visually presented large food quantity than the small food quantity in a choice task, particularly for quanities of lower ratio (i.e. a large difference between the two quantities) (Petrazzini and Wynne, 2016, 2017). This result conforms with Weber's law (Fechner, 1860), where two comparable quantities (i.e. when the small quantity divided by the large quantity produces a large value) are harder to discriminate 
than two different quantities (i.e. when the small quantity divided by the large quantity produces a small value). This ratio effect has also been found in chimpanzees (Beran, 2001) and pigeons (Roberts, 2010) during visual quantity discrimination and is suggestive for an approximate number system (ANS) (Feigenson et al., 2004). The ANS is a cognitive representation system of numbers larger than four, allowing a spontaneous approximation of number of objects (Piazza, 2011) without relying on language or symbolic representations. The Weber fraction in the ratio effect is the just-noticeable difference in stimulus intensity and can be derived from the accuracy performance as the difference between the two closest discriminable numerosities (Piazza, 2011). The Weber fraction is relatively constant and a fixed proportion of the reference sensory level. It can be described as follows, where $I$ is the original intensity of the stimulus and $\Delta I$ is the additional intensity to generate a noticeable difference (Equation 1):

$$
\frac{\Delta I}{I}=k
$$

Due to the constancy of the Weber fraction, numerical differences at higher ratio (i.e. when the small quantity divided by the large quantity produces a large value) are typically perceived with lower accuracy. This generally accounts for the reported decline in discrimination performance with decreasing difference between quantities (Petrazzini and Wynne, 2016, 2017) and, in particular, for the reliance on the total amount rather than on the number for discriminating quantities (Petrazzini and Wynne, 2016). However, this interpretation is inconsistent with the view in the literature (Agrillo and Beran, 2013) that for numbers smaller than four a different numerical system than the ANS is engage in the numerical representation, that is the object tracking system (OTS), wherein each perceived object is represented as a symbol, as a distinct unit, that adheres to the spatio-temporal principles of cohesion (Piazza, 2011): That is, such unit moves as a bounded whole and on connected unobstructed paths, principles that are conceptually related to the Gestalt laws in psychology (Ginsburg and Nicholls, 1988). Critically, the OTS is not affected by numerical ratio, i.e. 1:4 units can be discriminated with similar accuracy as 3:4 units. Therefore, the two-system explanation leads to a discrepancy between predicted and empirical outcomes, not only dog visual discrimination of quantities (Prato-Previde et al., 2008; Petrazzini and Wynne, 2016, 2017) but also in, for example, 
sea lions (Abramson et al., 2011), chimpanzees (Beran et al., 2008), and crows (Bogale et al., 2014). In response to that, a growing number of authors argue in favour of a single approximate number system that accounts for the complete number stream (Cantlon and Brannon, 2006; Cordes and Brannon, 2009; Beran and Parrish, 2016; Beran, 2007; Aulet et al., 2019).

Fewer studies addressed dogs' capabilities of olfactorily discriminating quantities: On average dogs paid more attention to a covered plate baited with more food (5 units) then one baited with less food (1 unit), but did not select that plate significantly more frequently (Horowitz et al., 2013). In contrast, in a more recent study dogs were able to reliably discriminate five hot dog slices from one hot dog slice (Jackson et al., 2021). In a separate experiment, quantity comparisons with varying Weber fractions showed that dogs did not adhere to the ratio effect, i.e. with increasing Weber fraction performance did not deteriorate. Jackson and colleagues (Jackson et al., 2021) suggest a difference in the processing of quantity discrimination between olfactory and visual domains. It might, however, also suggest that the assessment of olfactory capabilities in dogs is subjected to such large inter-breed variability, where a simplified general model of dog olfaction does not apply rather than a breed-specific one. For some breeds with greater olfactory sensitivity, ratio differences (e.g. 1:5 vs 3:5) might well be perceived and proportionally well differentiated, whereas breeds with lower sensitivity might hardly be capable of telling apart the largest of a difference between quantities and would be indifferent to any modulation in Weber fraction.

In order to gain more consensus on dogs' capabilities in olfactory discrimination of quantities and whether discrimination of quantities in the olfactory domain adheres to similar principles as the visual domain, we re-assess discrimination capabilities in a two-alternative forced choice (2AFC) paradigm. We also assess the detection rates of an olfactory food source, when the alternative was none in a 2AFC task. We hypothesise that dogs are better in detecting a food source than discriminating a larger from a smaller food source (Hypothesis 1). In this context, the detection task not only serves the purpose of replication of previous findings (Polgár et al., 2016), but it offers a means by which we can individually contrast detection scores of subjects and breeds with discrimination scores. Further, we hypothesise that dog olfactory capabilities vary largely between breeds, given the variability between breeds in a natural detection task (Polgár et al., 
2016) and the olfactory receptor sequence polymorphism between breeds (Tacher et al., 2005) (Hypothesis 2). More specifically we predict a correlation of performance scores with the skull form (dolicho- to brachycephalic) of the dog breeds, following the general assumption that those breeds with a brachycephalic skull (i.e. the length of the craniumis shorter than the width) are worse in olfactory detection than breeds with mesocephalic or dolichocephalic skulls (Polgár et al., 2016) (Hypothesis 3). We also hypothesise that Weber's law applies to dogs' olfactory discrimination in general; however, we expect that with the chosen Weber fractions some but not all breeds exhibit sufficient sensitivity to discriminate larger differences between quantities better than smaller ones (Hypothesis 4). For this reason, we chose a rather conservative experimental approach, where plates baited with food rewards were completely covered by a cup.

\section{Materials and methods}

\section{Subjects}

We used 41 domestic dogs (Canis lupus familiaris) (age in month: $\mu=21.13, \sigma=28.22$ ) of the following breeds: Miniature Australian Shepherd (MAS), White Swiss Shepherd (WS), Jack Russel Terrier (JR), Siberian Husky (SH), Spitz (SP), Golden Retriever (GR), Bichon Bolognese (BB), French Bulldog (FB) (Table 1). All dogs were owned and raised by dog shelters in Switzerland and France. The dogs were naïve to the tasks and scientific procedures. Dogs were not fed within three hours prior to the experiment.

\section{Apparatus}

All dogs were tested in a comparable fashion in enclosed and sheltered outdoor testing arena, measuring approximately $20-40 \mathrm{~m}^{2}$. For the experiments the dogs were separated from other dogs and had no visual or acoustic contact. Water was always available during the experiment.

\section{Procedure}

Dogs were tested in two tasks: (1) detection task, (2) discrimination task. In both of these tasks, we used a two-alternative forced choice (2AFC) paradigm, where (1) a food-reward of varying quantity was placed below one of two cups at $1 \mathrm{~m}$ distance, requiring the dog 
to indicate which of the cups contains the bait by approaching the respective cup, or (2) two food-rewards of varying quantities and reward ratios were placed below both cups at $1 \mathrm{~m}$ distance, requiring the dog to indicate its preference by approaching the cup, assuming it would naturally choose the cup with the strongest odour and therfore the larger quantity of food-reward.

Prior to the testing procedure, dogs underwent a habituation phase, where they were familiarised with items used in the experiments, i.e. cups and glass plates, and where they could freely explore the testing environment. They were also habituated to the human experimenters. After that, each dog was trained on the general task requirements by running four detection trials, where a food unit was placed under one of two cups and the dog was required to indicate which of the two cups it wants to get uncovered by approaching the cup of its choice.

In the testing phase, all dogs did both, the detection and the discrimination tasks. In both tasks, the dog's starting point was outside of the testing arena, kept at a leash by experimenter 1. Experimenter 2 placed a food reward under one cup (task 1) or both cups (task 2) according to the experimental protocol. The same experimenter then turned the cup upside-down onto a glass plate, such that the cups covered the food reward fully. The dog, guided by experimenter 1, entered the arena at the other end and was brought to the starting point of the trial, which was at a distance of $1.5 \mathrm{~m}$ to the midpoint of the cup arrangement. The dog was aligned equidistantly to the cups, forming a perpendicular bisector to the cup alignment. In the beginning of each trial, the dog was held back for $10 \mathrm{sec}$, allowing it to sense the reward location at the starting point, and then released by experimenter 1 to freely approach the target location. The dog then indicated its choice, and experimenter 2 lifted up both cups are simultaneously. The dog retrieved the food reward under the cup, if any. Experimenter 1 then led the dog out of the arena. Experimenter 2 exchanged the cups and glass plates and cleaned the environment, if necessary.

The reward-position was counter-balanced in the detection task and the larger reward-position was counter-balanced in the discrimination task. In the detection task, 1, 2 or 3 food reward units were used; in the discrimination task the following food reward units were compared: $1: 2 ; 1: 3 ; 1: 4$. Each reward unit was a standardised half-slice of Cervelat sausage, a typical Swiss sausage containing equal parts of beef, pork, bacon, and pork 
rind, along with spices, curing salt and cutter additives.

We further ensure that there was no experimenter-bias by having the Experimenter 2, which controlled the cup placement and the uncovering of the reward, looking down at the floor during the trial. Experimenter 1 was blind regarding the positioning of the food reward. In the detection task, each dog did three sessions on three different days, each containing 2 trials per quantity of food reward, hence 6 trials per session and a total of 18 trials per dog. In the discrimination task, each dog did three sessions on three different days, each containing 2 trials per food reward ratio $(1: 2,1: 3,1: 4)$, hence 6 trials per session and a total of 18 trials per dog. All experimental sessions were digitally recorded using two Panasonic ${ }^{\circledR}$ HC-V160 cameras (Panasonic ${ }^{\circledR}$ Corporation, Kadoma, Osaka, Japan).

\section{Data analysis}

Each trial resulted in either a correct detection (detection task), where correct was coded as '1' and incorrect as '0', or in a 'correct' discrimination (discrimination task), where '1' indicates large quantity of food reward and '0' small quantity of food reward. We are aware that for the discrimination task there is no 'correct' or 'incorrect' response. However, for simplicity, we refer to the responses as 'correct' or 'incorrect', assuming that dogs' natural choice would aim at maximising the quantity of food reward, hence they would prefer the larger quantity in the discrimination task over the smaller quantity (Ward and Smuts, 2007). Any outcome from the discrimination task needs to be discussed under this assumption. Henceforth, we refer to the response variable as performance (correct vs incorrect).

Analyses were performed in Matlab (Mathworks ${ }^{\circledR}$, Natick, Massachusetts, USA). We fitted a generalised linear mixed-effects models (fitglme function in Matlab) with binomial error structure and logit link function to our complete dataset (detection and discrimination task). We followed a model fitting procedure commonly accepted in the literature (Dobson and Barnett, 2018; Fedurek et al., 2019). To fully account for performance as response variable, we fitted four predictor variables: (1) breed of dog, (2) quantity of reward, (3) side of presentation, and (4) task (detection vs discrimination). Since we were interested in differentiating those effects that are similar across breeds from those that are different between breeds, we fitted the two-way interactions between breed and the remaining 
three main predictors. We also fitted the remaining interactions between factors task, side, and quantity. We assumed that there might be performance differences between breeds regarding discrimination of quantity of reward depending on the task. We also assumed that there might be a side response bias that depends on (1) breed and task, (2) breed and quantity, and (3) quantity and task. Hence, we fitted four additional three-way interactions. Thus, the full model contained six two-way interactions and four three-way interactions. In the next step, we fitted (1) random intercepts to subject, and random slopes and intercepts to the terms (2) side of presentation in subject, (3) task in subject and (4) quantity in subject, since we predicted that (1) individual dogs perform overall differently, (2) are potentially subject to a side bias, (3) perform better in one task than the other, and (4) some individuals' performance might depend on the presented quantity. We then created a null model, which consisted of the similar structure as the full model, however leaving only breed as fixed effect, while preserving all random effects. Using likelihood ratio test (LRT), we compared the null model with the full model. Assuming a significant improvement for the full model over the null model, the non-significant interaction terms were removed from the full model, reaching a model containing only significant interaction terms and both significant and non-significant main effects (Hector et al., 2010; Forstmeier and Schielzeth, 2011), henceforth referred to as the final model. Evaluation of fixed effect were on the basis of the final model.

This procedure resulted in the following models (Wilkinson notation):

Full model:

'Response $\sim 1+$ Breed + Quantity + Side + Task + Breed:Quantity + Breed:Side + Breed:Task + Side:Task + Quantity:Task + Quantity:Side + Breed:Side:Task + Breed:Quantity:Task + Breed:Quantity:Side + Quantity:Side:Task + (1|Subject $)+($ Side|Subject $)+($ Task|Subject $)+$ (Quantity|Subject)'

\section{Null model:}

'Response $\sim 1+$ Breed $+(1 \mid$ Subject $)+($ Side $\mid$ Subject $)+($ Task $\mid$ Subject $)+($ Quantity $\mid$ Subject $) '$

Final model: 
'Response $\sim 1$ + Breed + Quantity + Side + Task + Breed:Quantity + Breed:Task + Quantity:Task

+ Breed:Quantity:Task + (1|Subject $)+($ Side $\mid$ Subject $)+($ Task $\mid$ Subject $)+(\text { Quantity } \mid \text { Subject })^{\prime}$

In a second model, we fitted four predictor variables, replacing breed with cephalic index (CI), since we predicted that dogs's performance correlates with their skull form (dolicho- to brachycephalic). To this end, we determined the cephalic index for each breed (McGreevy et al., 2013; Teng et al., 2016; Evans and De Lahunta, 2013), ranked the breeds regarding the resulting cephalic index and binned them into three classes ('Cephalic index class', Table 1). We do not assign commonly known labels, such as brachy-, meso-, or dolichocephalic to individual breeds, rather than relative class labels, such as 'towards brachycephalic' (class 1), or 'towards dolichocephalic' (class 3).

This procedure resulted in the following models (Wilkinson notation):

Final model:

'Response $\sim 1+$ Cephalic index + Quantity + Side + Task + Cephalic index:Quantity + Cephalic index:Task + Quantity:Task + Cephalic index:Quantity:Task + (1|Subject) + $($ Side $\mid$ Subject $)+($ Task $\mid$ Subject $)+(\text { Quantity } \mid \text { Subject })^{\prime}$

In addition, we used Pearson correlation to determine the extent to which subjects performance scores in the detection and discrimination task were correlated.

\section{Results}

\section{Descriptive analysis}

Figure 1A-D shows the proportion correct responses over all dogs and breeds combined (A) and for each breed separately (B) in the detection task. Overall, dogs were correct in 70 to $75 \%$ of their choices (Figure 1A). We found a considerable variation between breeds, with Jack Russell Terriers, Siberian Huskies, and French Bulldogs showing relatively high performance scores and Bichon Bolognese showing relatively low performance scores that even fell below the chance level of $50 \%$ correct detection (Figure 1B). Factoring in the quantity of food reward, we found an overall increase of performance with increasing 
quantity of food (Figure 1C). At the level of individual breeds, however, there was little systematicity in terms of the predicted performance increase with increasing quantity of food reward (Figure 1D): The only breed that followed the prediction was the Siberian Husky.

Figure 1E-H shows the proportion correct responses over all dogs and breeds combined (E) and for each breed separately $(\mathrm{F})$ in the discrimination task. Overall, dogs were correct in $50 \%$ of their choices, thus, at chance level (Figure 1E). There was variation between breeds, with the same breeds performing marginally better as in the detection task (i.e., Jack Russell Terriers, Siberian Huskies, and French Bulldogs, while the latter showed large within-breed variation (Figure 1F). There were some breeds that performed below chance level, i.e. Miniature Australian Shepherd, Golden Retriever, and Bichon Bolognese. All the differences in the discrimination task were marginal. The quantity of food reward showed a small increase with increasing difference between the smaller and the larger quantities at the grand mean level (Figure $1 \mathrm{G}$ ). Also at the level of breeds, some breeds showed a systematic increase of performance with increasing difference between the smaller and the larger quantities: The breeds that followed the prediction most closely were the Siberian Husky and the French Bulldog (Figure 1H).

\section{Model investigation}

The full model, consisting of four fixed factors and their interactions that may explain the dogs' performance in the two tasks, was significantly different from the null model (LRT: $\chi_{\Delta 74}^{2}=154.51, p<0.001$; null model: $\mathrm{df}=20$, AIC $\left.=1436.6\right)$. In the final model, we found that breeds performed differently (LRT: $\chi_{\Delta 7}^{2}=27.84, p<0.001$; breed model: $\mathrm{df}=$ $55, \mathrm{AIC}=1438.3)$, and the two tasks yielded different overall results $\left(\mathrm{LRT}: \chi_{\Delta 1}^{2}=38.93\right.$ $p<0.001$; task model: $\mathrm{df}=61, \mathrm{AIC}=1461.4)$. These effects were not independent of each other, resulting in a significant interaction of breed and task (LRT: $\chi_{\Delta 7}^{2}=30.70$, $p<0.001$; breed-task model: $\mathrm{df}=55, \mathrm{AIC}=1441.1)$. Furthermore, we found a trend regarding the factor quantity (LRT: $\chi_{\Delta 2}^{2}=5.46, p=0.07$; quantity model: $\mathrm{df}=60$, $\mathrm{AIC}=1425.9)$. The interaction between factors breed and quantity was not significant, however close to a trend (LRT: $\chi_{\Delta 14}^{2}=21.16, p=0.10$; breed-quantity model: $\mathrm{df}=48$, $\mathrm{AIC}=1417.6)$. The factor side was not significant $\left(\mathrm{LRT}: \chi_{\Delta 1}^{2}=2.51, p=0.11\right.$; side model: $\mathrm{df}=61, \mathrm{AIC}=1424.9$ ). The remainig interactions were non-significant (Two-way 
interaction quantity and task: LRT: $\chi_{\Delta 2}^{2}=3.10, p=0.21$; quantity-task model: $\mathrm{df}=60$, $\mathrm{AIC}=1423.5$; three-way interaction breed, quantity and task: LRT: $\chi_{\Delta 14}^{2}=19.45, p=$ 0.15; breed-quantity-task model: $\mathrm{df}=48, \mathrm{AIC}=1415.9)$. The parameter estimates of the final model are summarised in the Table 2.

Random effects revealed that subjects performed at equal levels regarding the task involved and the quantity of food reward tested on: There was no significant interaction between the intercept subject and the slope grouped by task (LRT: $\chi_{\Delta 3}^{2}=2.36, p=0.50$; subject-task model: $\mathrm{df}=58, \mathrm{AIC}=1418.8)$, nor was there a significant interaction between the intercept subject and the slope grouped by quantity (LRT: $\chi_{\Delta 6}^{2}=1.97, p$ $=0.92 ;$ subject-quantity model: $\mathrm{df}=55, \mathrm{AIC}=1412.4)$. One Bichon was significantly different in its responses between the two tasks (see 'Subject x Task' in Table 3). Some subjects, however, showed a side bias, reflected in a correlation between the intercept grouped by subject and the slope grouped by side (LRT: $\chi_{\Delta 3}^{2}=80.88, p<0.001$; subject-side model: $\mathrm{df}=58, \mathrm{AIC}=1497.3$; see 'Subject $\mathrm{x}$ Side' in Table 3). Significantly different subjects were 4 Miniature Australian Shepherds, 2 French Bulldogs, 5 Bichons, and 1 Siberian husky. Adding the random factor subject with its interactions side improved the model, and subject variability in this regard cannot be neglected. There was, however, a deterioration in modelling the interaction between the intercept subject and the slope quantity and task (see above) as opposed to omitting these factors from the model, suggesting that there was no systematic difference regarding the discrimination performance as a function of quantity or task at the subject level.

The second model, modelling the factor cephalic index and replacing the factor breed, showed a significant modulation by the factor cephalic index (LRT: $\chi_{\Delta 2}^{2}=7.41, p<$ 0.05; cephalic index model: $\mathrm{df}=30, \mathrm{AIC}=1413.1$ ). In more detail, cephalic index class 1 was significantly different from cephalic index class 3 (Table 4; Figure 2A). As expected from the previous model, the factor task was significant (LRT: $\chi_{\Delta 1}^{2}=29.02$, $p<0.001 ;$ task model: $\mathrm{df}=31, \mathrm{AIC}=1436.7$; Table 4$)$ as well as the interaction of cephalic index and task (LRT: $\chi_{\Delta 2}^{2}=14.37, p<0.001$; task model: $\mathrm{df}=30$, AIC $=$ 1420; Table 4; Figure 2B). This interaction manifested in an increase of performance scores with increasing cephalic index class, i.e. from brachycephalic to dolichocephalic, in the discrimination task, but remains at similar levels across cephalic index classes in the discrimination task. Remaining factors follow the same directions as in the first 
model based on the factor breed. The parameter estimates of the model based on the factor cephalic index are summarised in the Table 4.

We further found that the detection and discrimination tasks correlated at the subject level $(r(39)=0.48, p<0.01$; Figure $2 \mathrm{C})$.

\section{Discussion}

In two experiments we studied the olfactory capabilities of untrained domestic dogs of different breeds, using a two-alternative forced choice (2AFC) paradigm. We hypothesised that, in general, dogs perform better in the detection of an olfactory cue, i.e. when no cue is the alternative choice, than in the discrimination of a larger olfactory cue from a smaller one (Hypothesis 1). Further, we hypothesised that given the genetic diversity of the canine olfactory system (Robin et al., 2009), dog breeds vary in olfactory capability, similar to a naturally occuring variance in visual capacity between breeds (McGreevy et al., 2004) (Hypothesis 2). We also predicted an influence on performance scores in both tasks depending on the skull form of the dog breed, i.e. dolicho-, meso- or brachycephalic, as a result of selective breeding causing functional anatomical changes in the brain (Hypothesis 3). Lastly, we also hypothesised that Weber's law applies to dogs' olfactory discrimination in general; however, due to the conservatively chosen Weber fractions some but not all breeds exhibit sufficient sensitivity to discriminate larger differences between quantities better than smaller ones, and hence, not all breeds might adhere to Weber's law under the given experimental circumstances (Hypothesis 4).

Addressing Hypothesis 1, we found that the detection and discrimination tasks produced largely different outcomes: While most breeds performed relatively well in detecting an olfactory stimulus, the discrimination of two olfactory stimuli varying in intensity (one being a larger quantity of food reward than the other) yielded about $50 \%$ correct responses - paralleling performance at chance level. This performance difference comes at no surpise, given the differences in computational demands between detection and discrimination. While in the discrimination task, subjects are required to discriminate the qualities of stimuli identities, i.e. the magnitudes of two smells, in the detection task, subjects are required to simply detect the presence of an identity, the smell, irrespective 
of the quality of the identity, i.e. the magnitude of smell (Schöpper et al., 2020). The relatively low discrimination performance is in accordance with Horowitz and colleagues (Horowitz et al., 2013), who found that dogs chose the larger quantity of food reward more often $(61 \%)$, but not significantly more than the smaller quantity. Dogs in their study were given the chance to explore two covered plates for about 3 seconds each, either in sequential or in simultaenous presentation. This procedure is different from ours, where dogs were relying on sniffing the larger quantity from $1 \mathrm{~m}$ distance and then indicating the cup of choice by approaching it. Our methodological approach resulted in higher task difficulty, given our intention to amplify and map out inter-breed differences. This more conservative approach might, thus, explain the even lower average performance scores during the detection and discrimination tasks in our study than in Polgár et al. (2016) and Horowitz et al. (2013), respectively. Notably, the rather low olfactory capability in our study and the study by Horowitz et al. (2013) is in contrast to dogs' visual capability in discriminating two food quantities (Prato-Previde et al., 2008; Petrazzini and Wynne, 2016; Ward and Smuts, 2007). This contrast between sensory modalities does not necessarily suggest that dogs discriminate quantities predominantly relying on vision or that neural mechanisms for olfactory discrimination do no exist, rather than that the visual and the olfactory tasks are by no means comparable with regard to the necessary magnitude of stimulation to generate a just-noticeable-difference. In other words, the Weber fraction varies between sensory modalities. Along the same lines, it might be that in a more naturalistic scenario where visual and olfactory stimulation co-occur the information from both modalities converge into a multisensory representation, potentially leading to multisensory enhancement, generating a more coherent representation based on which a more well-informed decision can be taken (Mercier and Cappe, 2020; Franzen et al., 2020). This redundancy principle, i.e. acquiring overlapping information from the environment, is a well-known design principles of intelligent systems and leads to systemic robustness and behavioural diversity (Pfeifer et al., 2005). In other words, the decision is made on the basis of multisensory rather than unisensory representations, leaving unisensory testing methods with little ecological relevance. This said, an explanation for why visual discrimination appears to be more sensitive than olfactory discrimination might be that the two modalities are complementary in the sense that, while the olfactory domain provides directional information of a (larger) prey in a very coarse manner, the 
visual domain provides the means to spot the (larger) prey at very high spatial and temporal resolution. Hence, there is no evolutionary pressure for an olfactory system with higher spatial resolution given the high precision of the visual system. Another explanation, which is not mutually exclusive to the above, is that dog olfaction does not comply with the notion of two systems, i.e. the ANS and OTS, as suggested in the visual domain, but rather relies on an all-or-nothing response system (Jackson et al., 2021). While in most sensory modalities the underlying physical principles are well understood; in olfaction, it remains unknown what principles specific chemical compounds in the air, forming odours, adhere to (Pannunzi and Nowotny, 2019). Hence, the lack of knowledge regarding the physical principles of odours prevents us from prematurely conclude that the olfactory system underlies similar cognitive mechanisms as the visual system regarding numerosity. Also, the fact that, in our experiment and in Horowitz et al. (2013), the olfactory stimuli were covered during presentation further augments the inability to precisely control the odours experimentally and to infer physical properties like concentration and spatio-temporal distribution.

Addressing Hypothesis 2, we found that detection and discrimination performances also varied largely between breeds. In detail, Jack Russell Terriers performed best in the detection task, followed by French Bulldogs, Siberian Huskies and White Swiss Shepherd. French Bulldogs and Jack Russell Terriers also performed best in the discrimination task, followed closely by Siberian Huskies and White Swiss Shepherd. When directly comparing olfactory detection and discrimination performances, individual subjects were positively correlated, suggesting that those individuals that were better in the detection task were also better in the discrimination task. This supports the notion that, in theory, dogs possess the capability of discrimination of olfactorily presented quantities, however, in our experiment the requirements for the discrimination task were too high, i.e. the differences between two presented olfactory cues were too small. This directly highlights the additional task demands, when synchronously evaluating two olfactory sources as opposed to localising one: While keeping the ratio constant between tasks (i.e. we presented single cues at magnitudes of 1,2, and 3 in the detection and two cues at magnitudes of 1 and 2, 1 and 3, and 1 and 4 in the discrimination task), the performance scores dropped about $25 \%$ between the detection and discrimination tasks. 
It needs to be noted that we did not aim at highest performance scores rather than at detectable differences between the two tasks and breeds. Also, systematically adapting the sensitivity range, potentially leads to a decrease of the Weber fraction to a degree where mathematically a discrimination task approximates a detection task, i.e. when the smaller quantity is marginally small and fully overshadowed by the large quantity, and, hence, conceptually it becomes increasingly difficult to distinguish a discrimination task from a detection task. This is particularly problematic without a solid understanding regarding the spatio-temporal distribution of odours (Pannunzi and Nowotny, 2019).

Addressing Hypothesis 3, we found that dogs of different cephalic index classes performed differently overall as well as a function of the task. We are aware that our classification of dog breeds into three classes does not follow the scientifically accepted classification of breeds into brachy-, meso- and dolichocephalic types of breeds, but given the limited number of breeds used in our study and the relatively centralised, i.e. mesocephalic, distribution, we decided to proportionally scale them within the pool of breeds used in this study. Clearly, a more detailed assessment on how cephalic type influences the olfactory detection and discrimination performances is required. Nevertheless, our analyses revealed that dog breeds differed in their abilities to detect and discriminate olfactory cues not arbitrarily, but in accordance with the general assumption that those breeds with a brachycephalic skull (i.e. the length of the cranium is shorter than the width) are worse in olfactory detection than breeds with mesocephalic or dolichocephalic skulls (Polgár et al., 2016), but see (Hall et al., 2015). It has been argued that selective breeding for reduced faces not only resulted in functional anatomical changes in the nose, but in a reduction in brain matter devoted to olfaction and spatial reorganisation of the brain (Selba et al., 2021). While a reduction of olfactory cortical areas clearly impacts the associated functions, such as olfactory detection and discrimination, the impact of spatial reorganisation of the brain areas and associated functions are currently investigated: Anatomical differences between breeds as a result of selective breeding and reflected in the cepahlic index have been reported (Selba et al., 2021; Hecht et al., 2019).

Addressing Hypothesis 4, we found that statistically dogs did not adhere to the ratio effect, i.e. their discrimination performance did not improve with decreasing Weber 
fraction or increasing differences between the quantities presented. Nevertheless, the factor quantity played a crucial role in the model fitting and cannot be ignored due to its $p$-value being close to significance (7\%). Indidividual dog breeds, such as the Siberian Husky, indeed reflect this trend very clearly: While trials with small quantity differences yielded lower correct discrimination, those trials with larger quantity difference yielded

higher correct discrimination. This finding in the olfactory domain parallels findings in the visual domain (Petrazzini and Wynne, 2016, 2017) and suggests similar underlying principles for the discrimination of two magnitudes in the visual and the olfactory sensory modalities. The finding support the idea of an olfactory equivalent to the approximate number system (ANS) (Feigenson et al., 2004) in the visual domain, which due to the nature of olfactory stimulation most likely adheres to an approximation of olfactory intensity throughout the complete intensity scale, and is therefore in contrast to the idea of two number systems, the ANS for numbers larger than four and the object tracking system (OTS) for numbers smaller than four as proposed in the visual domain.

Furthermore, it turned out that subject as random factor does not improve the model-fitting. This suggests inter-individual performance differences do no systematically occur neither at the level of task-related performances nor in dependence on food quantity. However, a limited number of subjects showed side-biases that led to a significant interaction of subject and side. This side-bias can be interpreted as a coping strategy for increasingly difficult task requirements and might also reduce with less conservative experimental methods.

\section{Acknowledgements}

We are grateful for the Startup-funding of Taipei Medical University (108-6402-004-112) and the Taiwan Ministry of Science and Technology research grant (110-2311-B-038-002) awarded to CDD. We thank Guillaume Dezecache, Ph.D., for comments on the manuscript. 


\section{Author contributions}

EF: study design, data collection, acquisition of resources; CDD: study design, analysis and interpretation, writing article.

\section{Funding}

This study was funded via the Ambizione Fellowship of the Swiss National Science Foundation (SNSF) (PZ00P3_154741), the Startup-funding of Taipei Medical University (108-6402-004-112) and the Taiwan Ministry of Science and Technology (110-2311-B-038-002) awarded to CDD.

\section{Conflict of interest}

The authors declare that they have no conflict of interest. The authors have no affiliations with or involvement in any organization or entity with any financial interest, or non-financial interest in the subject matter or materials discussed in this manuscript.

\section{Ethical approval}

All applicable international, national, and/or institutional guidelines for the care and use of animals were followed, i.e., the Swiss law on animal protection and welfare. This study was approved by the Swiss Federal Veterinary Office (approval number VD3383) for experiments conducted in Switzerland. According to the local authorities (Comité d'Ethique de l'Expérimentation Animale Grand Campus Dijon, Université de Bourgogne, Maison de l'Université, Esplanade Erasme, 21078 Dijon, France), non-invasive studies on dogs are allowed to be conducted without any special permission in France. 


\section{${ }_{522}$ Tables}

${ }_{523}$ Table 1: Dog breeds used in the study and assigned cephalic index class.

\begin{tabular}{lccc}
\multicolumn{1}{c}{$\begin{array}{c}\text { Dog } \\
\text { breed }\end{array}$} & Abbreviation & $\begin{array}{c}\text { \# of } \\
\text { individuals }\end{array}$ & $\begin{array}{c}\text { Cephalic } \\
\text { index class }\end{array}$ \\
\hline Miniature Australian Shepherd & MAS & 9 & 3 \\
White Swiss Shepherd & WS & 3 & 3 \\
Jack Russell & JR & 2 & 2 \\
Siberian Husky & SH & 9 & 3 \\
Spitz & SP & 1 & 1 \\
Golden Retriever & GR & 5 & 2 \\
Bichon Bolognese & BB & 9 & 1 \\
French Bulldog & FB & 3 & 1 \\
\hline Total & & 41 &
\end{tabular}


bioRxiv preprint doi: https://doi.org/10.1101/2022.02.04.479113; this version posted February 7, 2022. The copyright holder for this preprint (which was not certified by peer review) is the author/funder, who has granted bioRxiv a license to display the preprint in perpetuity. It is made available under aCC-BY-NC 4.0 International license.

Table 2: Results of the model investigating performance scores in detection and discrimination

tasks. The table contains parameter estimates for the final model based on the factor 'breed' (first model).

\begin{tabular}{|c|c|c|c|c|c|c|}
\hline & Estimate & SE & $t$-stat & DF & $p$-value & $\begin{array}{c}\text { CI }(95 \%) \\
\text { [lower, upper] }\end{array}$ \\
\hline Intercept & 0.48 & 0.10 & 4.88 & 1185 & 0.001 & {$\left[\begin{array}{lll}0.29 & 0.67\end{array}\right]$} \\
\hline Miniature Australian Shepherd (MAS) & -0.05 & 0.15 & -0.31 & 1185 & 0.76 & {$\left[\begin{array}{ll}-0.35 & 0.25\end{array}\right]$} \\
\hline White Swiss Shepherd (WS) & 0.14 & 0.24 & 0.59 & 1185 & 0.55 & {$\left[\begin{array}{ll}-0.33 & 0.61\end{array}\right]$} \\
\hline Jack Russell (JR) & 0.41 & 0.32 & 1.28 & 1185 & 0.20 & {$[-0.221 .04]$} \\
\hline Siberian Husky (SH) & 0.35 & 0.17 & 2.03 & 1185 & 0.04 & {$\left[\begin{array}{ll}0.01 & 0.69\end{array}\right]$} \\
\hline Spitz (SP) & -0.09 & 0.41 & -0.23 & 1185 & 0.82 & {$\left[\begin{array}{ll}-0.89 & 0.71]\end{array}\right.$} \\
\hline Golden Retriever (GR) & -0.30 & 0.21 & -1.47 & 1185 & 0.14 & {$\left[\begin{array}{ll}-0.71 & 0.10\end{array}\right]$} \\
\hline Bichon Bolognese (BB) & -0.80 & 0.17 & -4.75 & 1185 & 0.001 & {$[-1.13-0.47]$} \\
\hline \multicolumn{7}{|l|}{ (against French Bulldog (FB)) } \\
\hline Quantity $\Delta 1$ (1 unit) & -0.10 & 0.09 & -1.19 & 1185 & 0.23 & {$\left[\begin{array}{ll}-0.27 & 0.07\end{array}\right]$} \\
\hline Quantity $\Delta 2$ (2 units) & -0.07 & 0.08 & -0.84 & 1185 & 0.40 & {$\left[\begin{array}{ll}-0.23 & 0.09\end{array}\right]$} \\
\hline \multicolumn{7}{|l|}{ (against Quantity $\Delta 3$ (3 units)) } \\
\hline Right & -0.16 & 0.10 & -1.61 & 1185 & 0.11 & {$\left[\begin{array}{ll}-0.34 & 0.03\end{array}\right]$} \\
\hline \multicolumn{7}{|l|}{ (against Left) } \\
\hline Detection task & 0.43 & 0.06 & 6.92 & 1185 & 0.001 & {$\left[\begin{array}{ll}0.31 & 0.55\end{array}\right]$} \\
\hline \multicolumn{7}{|l|}{ (against Discrimination task) } \\
\hline Miniature Australian Shepherd (MAS) : Detection & 0.07 & 0.09 & 0.79 & 1185 & 0.43 & {$\left[\begin{array}{ll}-0.11 & 0.26\end{array}\right]$} \\
\hline White Swiss Shepherd (WS) : Detection & 0.08 & 0.14 & 0.53 & 1185 & 0.60 & {$\left[\begin{array}{ll}-0.21 & 0.36\end{array}\right]$} \\
\hline Jack Russell (JR) : Detection & 0.25 & 0.23 & 1.09 & 1185 & 0.28 & {$[-0.200 .70]$} \\
\hline Siberian Husky (SH) : Detection & 0.22 & 0.12 & 1.79 & 1185 & 0.07 & {$\left[\begin{array}{ll}-0.02 & 0.45\end{array}\right]$} \\
\hline Spitz (SP) : Detection & -0.06 & 0.26 & -0.22 & 1185 & 0.82 & {$\left[\begin{array}{ll}-0.57 & 0.45\end{array}\right]$} \\
\hline Golden Retriever (GR) : Detection & -0.02 & 0.12 & -0.13 & 1185 & 0.90 & {$\left[\begin{array}{ll}-0.25 & 0.22\end{array}\right]$} \\
\hline Bichon Bolognese (BB): Detection & -0.55 & 0.11 & -4.99 & 1185 & 0.001 & {$[-0.77-0.33]$} \\
\hline \multicolumn{7}{|l|}{ (against French Bulldog (FB) and Discrimination task) } \\
\hline Miniature Australian Shepherd (MAS) : Quantity $\Delta 1$ (1 unit) & 0.14 & 0.13 & 1.08 & 1185 & 0.28 & {$[-0.110 .40]$} \\
\hline White Swiss Shepherd (WS) : Quantity $\Delta 1$ (1 unit) & -0.10 & 0.20 & -0.46 & 1185 & 0.64 & {$[-0.480 .30]$} \\
\hline Jack Russell (JR) : Quantity $\Delta 1$ (1 unit) & 0.10 & 0.30 & 0.34 & 1185 & 0.74 & {$\left[\begin{array}{ll}-0.49 & 0.69\end{array}\right]$} \\
\hline Siberian Husky (SH) : Quantity $\Delta 1$ (1 unit) & -0.31 & 0.16 & -1.93 & 1185 & 0.05 & {$[-0.620 .01]$} \\
\hline Spitz (SP) : Quantity $\Delta 1$ (1 unit) & 0.07 & 0.37 & 0.19 & 1185 & 0.85 & {$[-0.650 .79]$} \\
\hline Golden Retriever (GR) : Quantity $\Delta 1$ (1 unit) & 0.18 & 0.17 & 1.07 & 1185 & 0.29 & {$[-0.150 .52]$} \\
\hline Bichon Bolognese (BB): Quantity $\Delta 1$ (1 unit) & 0.03 & 0.15 & 0.20 & 1185 & 0.84 & {$[-0.27 \quad 0.33]$} \\
\hline Miniature Australian Shepherd (MAS) : Quantity $\Delta 2$ (2 units) & 0.21 & 0.12 & 1.67 & 1185 & 0.10 & {$\left[\begin{array}{lll}-0.04 & 0.45\end{array}\right]$} \\
\hline White Swiss Shepherd (WS) : Quantity $\Delta 2$ (2 units) & 0.08 & 0.19 & 0.45 & 1185 & 0.66 & {$\left[\begin{array}{ll}-0.29 & 0.45]\end{array}\right]$} \\
\hline Jack Russell (JR) : Quantity $\Delta 2$ (2 units) & 0.10 & 0.29 & 0.33 & 1185 & 0.74 & {$\left[\begin{array}{ll}-0.48 & 0.67\end{array}\right]$} \\
\hline Siberian Husky (SH) : Quantity $\Delta 2$ (2 units) & -0.07 & 0.15 & -0.46 & 1185 & 0.65 & {$\left[\begin{array}{ll}-0.37 & 0.23\end{array}\right]$} \\
\hline Spitz (SP) : Quantity $\Delta 2$ (2 units) & -0.32 & 0.34 & -0.95 & 1185 & 0.34 & {$\left[\begin{array}{ll}-0.99 & 0.35\end{array}\right]$} \\
\hline Golden Retriever (GR) : Quantity $\Delta 2$ (2 units) & -0.09 & 0.16 & -0.57 & 1185 & 0.57 & {$\left[\begin{array}{ll}-0.40 & 0.22\end{array}\right]$} \\
\hline Bichon Bolognese (BB): Quantity $\Delta 2$ (2 units) & -0.01 & 0.15 & -0.07 & 1185 & 0.94 & {$\left[\begin{array}{ll}-0.30 & 0.28\end{array}\right]$} \\
\hline
\end{tabular}


${ }_{529}$ Table 3: Individual performance scores for the detection and discrimination tasks. The

530 table contains proportion correct responses, the intercepts of the random factor subject

${ }_{531}$ and slopes grouped by side and task, and the ratio between the tasks:

${ }_{532}$ ratio $=\frac{\text { discrimination }}{\text { detection }}$

\begin{tabular}{|c|c|c|c|c|c|c|}
\hline Breed & $\begin{array}{c}\text { Individual } \\
{[\#]}\end{array}$ & Detection & Discrimination & Ratio & $\begin{array}{l}\text { Subject } \mathrm{x} \\
\text { Side }\end{array}$ & $\begin{array}{c}\text { Subject } \mathrm{x} \\
\quad \text { Task }\end{array}$ \\
\hline \multirow[t]{9}{*}{ Miniature Australian Shepherd (MAS) } & 1 & 0.83 & 0.5 & 0.60 & & \\
\hline & 2 & 0.67 & 0.22 & 0.33 & * & \\
\hline & 3 & 0.78 & 0.39 & 0.50 & * & \\
\hline & 4 & 0.89 & 0.61 & 0.69 & & \\
\hline & 5 & 0.83 & 0.56 & 0.67 & & \\
\hline & 6 & 0.44 & 0.33 & 0.75 & $* * *$ & \\
\hline & 7 & 0.72 & 0.44 & 0.62 & $* * *$ & \\
\hline & 8 & 0.94 & 0.50 & 0.53 & & \\
\hline & 9 & 0.89 & 0.44 & 0.50 & & \\
\hline \multirow[t]{3}{*}{ White Swiss Shepherd (WS) } & 1 & 0.83 & 0.61 & 0.73 & & \\
\hline & 2 & 0.83 & 0.50 & 0.60 & & \\
\hline & 3 & 0.89 & 0.50 & 0.56 & & \\
\hline \multirow[t]{2}{*}{ Jack Russell (JR) } & 1 & 0.83 & 0.50 & 0.60 & & \\
\hline & 2 & 1.00 & 0.67 & 0.67 & & \\
\hline \multirow[t]{9}{*}{ Siberian Husky (SH) } & 1 & 1.00 & 0.33 & 0.33 & & \\
\hline & 2 & 0.92 & 0.50 & 0.55 & & \\
\hline & 3 & 0.83 & 0.58 & 0.70 & * & \\
\hline & 4 & 0.75 & 0.67 & 0.89 & & \\
\hline & 5 & 0.83 & 0.50 & 0.60 & & \\
\hline & 6 & 0.92 & 0.42 & 0.45 & & \\
\hline & 7 & 1.00 & 0.67 & 0.67 & & \\
\hline & 8 & 0.92 & 0.67 & 0.73 & & \\
\hline & 9 & 0.75 & 0.67 & 0.89 & & \\
\hline Spitz (SP) & 1 & 0.75 & 0.50 & 0.67 & & \\
\hline \multirow[t]{5}{*}{ Golden Retriever (GR) } & 1 & 0.78 & 0.44 & 0.57 & & \\
\hline & 2 & 0.72 & 0.33 & 0.46 & & \\
\hline & 3 & 0.61 & 0.44 & 0.73 & & \\
\hline & 4 & 0.72 & 0.39 & 0.54 & & \\
\hline & 5 & 0.78 & 0.61 & 0.79 & & \\
\hline \multirow[t]{9}{*}{ Bichon Bolognese (BB) } & 1 & 0.33 & 0.33 & 1.00 & & \\
\hline & 2 & 0.33 & 0.50 & 1.50 & * & \\
\hline & 3 & 0.50 & 0.42 & 0.83 & * & \\
\hline & 4 & 0.50 & 0.42 & 0.83 & $* * *$ & \\
\hline & 5 & 0.17 & 0.42 & 2.50 & $*$ & \\
\hline & 6 & 0.33 & 0.42 & 1.25 & $* * *$ & \\
\hline & 7 & 0.17 & 0.33 & 2.00 & & \\
\hline & 8 & 0.83 & 0.17 & 2.00 & & \\
\hline & 9 & 1.00 & 1.00 & 1.00 & & $* * *$ \\
\hline \multirow[t]{3}{*}{ French Bulldog (FB) } & 1 & 0.94 & 0.42 & 0.44 & * & \\
\hline & 2 & 0.67 & 0.92 & 1.38 & & \\
\hline & 3 & 0.94 & 0.50 & 0.53 & $*$ & \\
\hline
\end{tabular}

Significance level [\%]: $*=5, * *=1, * * *=.1$ 
'cephalic index' (second model).

\begin{tabular}{|c|c|c|c|c|c|c|c|}
\hline & & Estimate & $\mathrm{SE}$ & $t$-stat & $\mathrm{DF}$ & $p$-value & $\begin{array}{c}\mathrm{CI}(95 \%) \\
{[\text { lower, upper] }}\end{array}$ \\
\hline \multirow{4}{*}{ 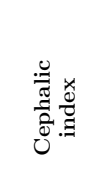 } & Intercept & 0.34 & 0.11 & 3.22 & 1199 & 0.01 & {$\left[\begin{array}{lll}0.13 & 0.55\end{array}\right]$} \\
\hline & Cephalic index class 1 & -0.33 & 0.14 & -2.33 & 1199 & 0.02 & {$[-0.60-0.05]$} \\
\hline & Cephalic index class 2 & 0.06 & 0.17 & 0.33 & 1199 & 0.74 & {$\left[\begin{array}{ll}-0.28 & 0.39\end{array}\right]$} \\
\hline & (against Cephalic index class 3) & & & & & & \\
\hline \multirow{3}{*}{ 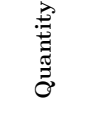 } & Quantity $\Delta 1$ (1 unit) & -0.07 & 0.08 & -0.92 & 1199 & 0.36 & {$\left[\begin{array}{ll}-0.21 & 0.08\end{array}\right]$} \\
\hline & Quantity $\Delta 2$ (2 units) & -0.05 & 0.07 & -0.71 & 1199 & 0.47 & {$\left[\begin{array}{lll}-0.18 & 0.09\end{array}\right]$} \\
\hline & (against Quantity $\Delta 3$ (3 units)) & & & & & & \\
\hline \multirow{2}{*}{ 总 } & Right & -0.15 & 0.10 & -1.61 & 1199 & 0.11 & {$\left[\begin{array}{lll}-0.34 & 0.03]\end{array}\right]$} \\
\hline & (against Left) & & & & & & \\
\hline \multirow{2}{*}{ 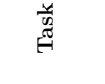 } & Detection task & 0.38 & 0.06 & 6.48 & 1199 & 0.001 & {$\left[\begin{array}{lll}0.26 & 0.49\end{array}\right]$} \\
\hline & (against Discrimination task) & & & & & & \\
\hline \multirow{5}{*}{ 总 } & Cephalic index class 1: Quantity $\Delta 1$ (1 unit) & -0.04 & 0.11 & -0.34 & 1199 & 0.73 & {$\left[\begin{array}{lll}-0.24 & 0.17\end{array}\right]$} \\
\hline & Cephalic index class $1:$ Quantity $\Delta 2$ (2 units) & -0.03 & 0.10 & -0.33 & 1199 & 0.74 & {$\left[\begin{array}{lll}-0.22 & 0.16\end{array}\right]$} \\
\hline & Cephalic index class 2: Quantity $\Delta 1$ (1 unit) & 0.11 & 0.12 & 0.91 & 1199 & 0.36 & {$\left[\begin{array}{ll}-0.13 & 0.35\end{array}\right]$} \\
\hline & Cephalic index class $2:$ Quantity $\Delta 2$ (2 units) & -0.07 & 0.11 & -0.69 & 1199 & 0.49 & {$\left[\begin{array}{lll}-0.29 & 0.14\end{array}\right]$} \\
\hline & (against Cephalic index class 3 and Quantity $\Delta 3$ (3 units)) & & & & & & \\
\hline \multirow{3}{*}{ 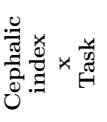 } & Cephalic index class 1 : Detection & -0.30 & 0.08 & -3.76 & 1199 & 0.001 & {$[-0.46-0.15]$} \\
\hline & Cephalic index class 2 : Detection & 0.12 & 0.09 & 1.31 & 1199 & 0.19 & {$\left[\begin{array}{lll}-0.06 & 0.31\end{array}\right]$} \\
\hline & (against Cephalic index class 3 and Discrimination task) & & & & & & \\
\hline \multirow{3}{*}{ 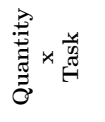 } & Quantity $\Delta 1$ (1 unit) : Detection & -0.05 & 0.07 & -0.69 & 1199 & 0.49 & {$\left[\begin{array}{lll}-0.17 & 0.08\end{array}\right]$} \\
\hline & Quantity $\Delta 2$ (2 units) : Detection & -0.01 & 0.07 & -0.10 & 1199 & 0.92 & {$\left[\begin{array}{ll}-0.14 & 0.12\end{array}\right]$} \\
\hline & (against Quantity $\Delta 3$ (3 units) and Discrimination task) & & & & & & \\
\hline
\end{tabular}


bioRxiv preprint doi: https://doi.org/10.1101/2022.02.04.479113; this version posted February 7, 2022. The copyright holder for this preprint (which was not certified by peer review) is the author/funder, who has granted bioRxiv a license to display the preprint in perpetuity. It is made available under aCC-BY-NC 4.0 International license.

\section{${ }_{537}$ Figures}

538 Figure 1:
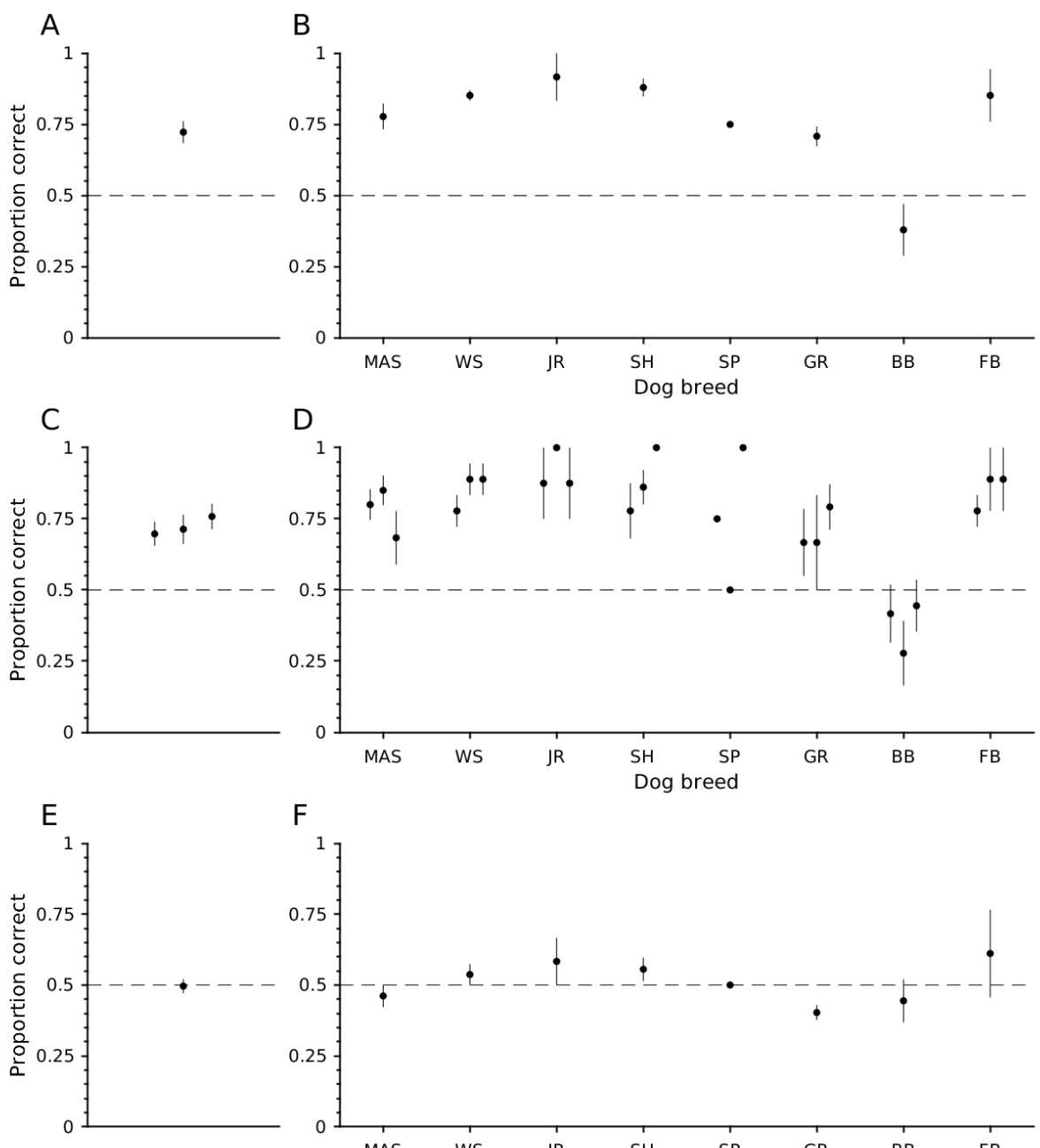

$\mathrm{F}$
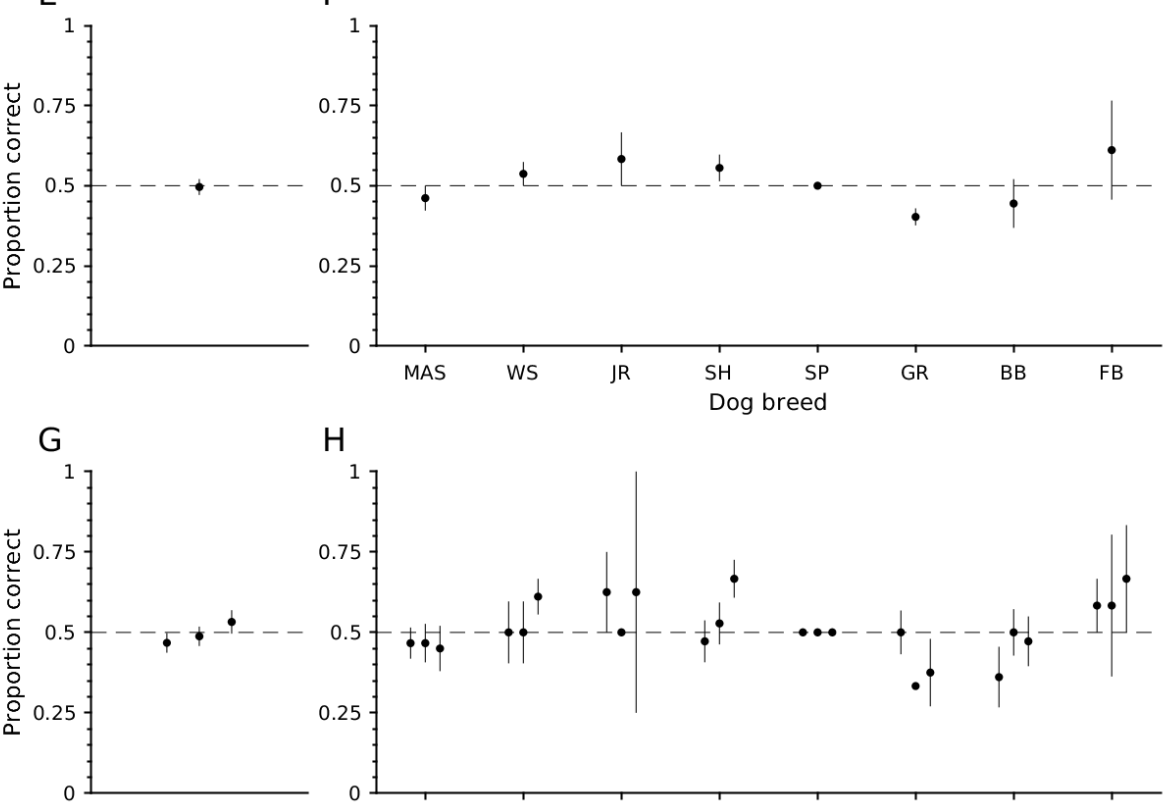

\section{$\mathrm{H}$}

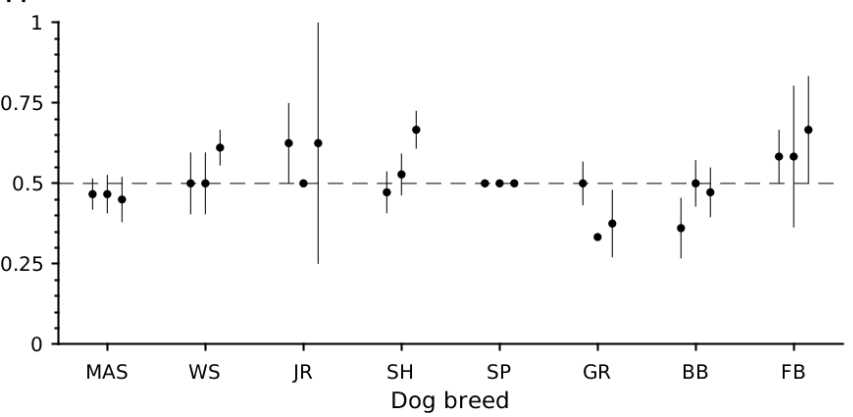


bioRxiv preprint doi: https://doi.org/10.1101/2022.02.04.479113; this version posted February 7, 2022. The copyright holder for this preprint (which was not certified by peer review) is the author/funder, who has granted bioRxiv a license to display the preprint in perpetuity. It is made available under aCC-BY-NC 4.0 International license.

540 Figure 2:

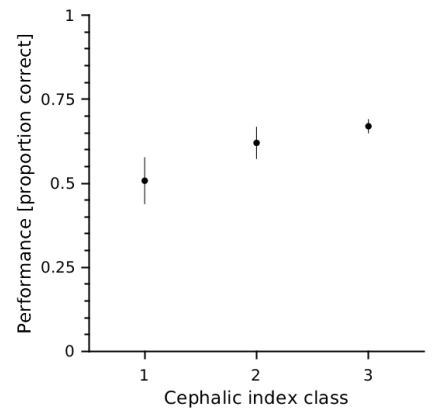

B

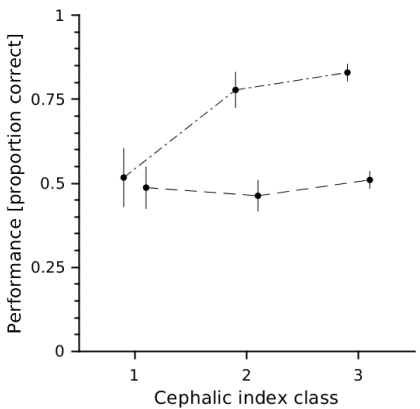

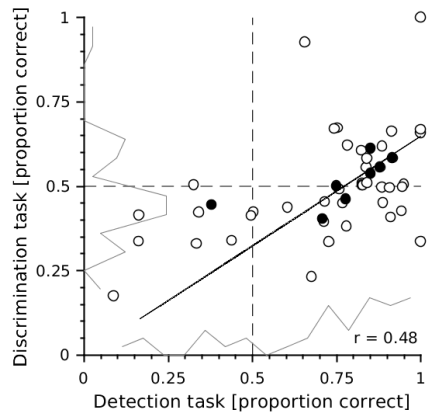




\section{Figure captions}

Figure caption 1: Detection and discrimination tasks. All panels show the proportion correct responses in the detection task on the y-axis. The black dots represent the mean and the whiskers the standard error of the mean. A-D. Detection task. A shows the overall performance (grand mean); B shows the performances for different breeds (x-axis); C and $\mathrm{D}$ are similar to $\mathrm{A}$ and $\mathrm{B}$, but show the performance as a function of quantity of food reward (C) for each breed (D). For each set of three means and standard errors of the mean, the smallest food reward ( 1 unit) is shown to the left and the largest ( 3 units) to the right. E-H. Discrimination task. E shows the overall performance (grand mean); F shows the performances for different breeds (x-axis); $\mathrm{G}$ and $\mathrm{H}$ are similar to $\mathrm{E}$ and $\mathrm{F}$, but show the performance as a function of quantity of food reward $(\mathrm{G})$ for each breed $(\mathrm{H})$.

Figure caption 2: Cepahlic index classes, and correlation of detection and discrimination performance scores. A. The general perfomance scores are shown as a function of the cephalic index classes. Class labels on the x-axis stand for 'towards brachycephalic' (class 1) and 'towards dolichocephalic' (class 3). Shown are the means and standard errors of the mean across individuals. B. Similar as in A, but for the detection task (dash-dotted line) discrimination task (dashed line), respectively. C. Open circles indicate individual subjects' performance in the detection task (x-axis) and the discrimination task (y-axis). The regression line (solid black line) was calculated on the basis of the individual subjects' performance scores. For illustration, filled black circles indicate the mean across subjects for each breed. The solid grey lines show the distribution of data samples, computed via histograms containing 20 equally large bins. The dashed black lines indicate the $50 \%$ chance level for both tasks. 


\section{References}

Abramson JZ, Hernández-Lloreda V, Call J, Colmenares F (2011) Relative quantity judgments in south american sea lions (otaria flavescens). Animal cognition 14(5):695

Agrillo C, Beran MJ (2013) Number without language: comparative psychology and the evolution of numerical cognition. Frontiers in Psychology 4:295

Aulet LS, Chiu VC, Prichard A, Spivak M, Lourenco SF, Berns GS (2019) Canine sense of quantity: evidence for numerical ratio-dependent activation in parietotemporal cortex. Biology letters 15(12):20190666

Becker RF, King JE, Markee J (1962) Studies on olfactory discrimination in dogs: Ii. discriminatory behavior in a free environment. Journal of comparative and physiological psychology 55(5):773

Beran MJ (2001) Summation and numerousness judgments of sequentially presented sets of items by chimpanzees (pan troglodytes). Journal of Comparative Psychology $115(2): 181$

Beran MJ (2007) Rhesus monkeys (macaca mulatta) enumerate large and small sequentially presented sets of items using analog numerical representations. Journal of Experimental Psychology: Animal Behavior Processes 33(1):42

Beran MJ, Parrish AE (2016) Going for more: Discrete and continuous quantity judgments by nonhuman animals. In: Continuous Issues in Numerical Cognition, Elsevier, pp 175-192

Beran MJ, Evans TA, Harris EH (2008) Perception of food amounts by chimpanzees based on the number, size, contour length and visibility of items. Animal Behaviour 75(5):1793-1802

Bidder OR, Di Virgilio A, Hunter JS, McInturff A, Gaynor KM, Smith AM, Dorcy J, Rosell F (2020) Monitoring canid scent marking in space and time using a biologging and machine learning approach. Scientific reports 10(1):1-13

Bogale BA, Aoyama M, Sugita S (2014) Spontaneous discrimination of food quantities in the jungle crow, corvus macrorhynchos. Animal Behaviour 94:73-78 
Bräuer J, Blasi D (2021) Dogs display owner-specific expectations based on olfaction. Scientific Reports 11(1):1-10

Cablk ME, Sagebiel JC, Heaton JS, Valentin C (2008) Olfaction-based detection distance: a quantitative analysis of how far away dogs recognize tortoise odor and follow it to source. Sensors 8(4):2208-2222

Cafazzo S, Natoli E, Valsecchi P (2012) Scent-marking behaviour in a pack of free-ranging domestic dogs. Ethology 118(10):955-966

Cantlon JF, Brannon EM (2006) Shared system for ordering small and large numbers in monkeys and humans. Psychological science 17(5):401-406

Cordes S, Brannon EM (2009) Crossing the divide: Infants discriminate small from large numerosities. Developmental psychology 45(6):1583

Dobson AJ, Barnett AG (2018) An introduction to generalized linear models. CRC press Evans HE, De Lahunta A (2013) Miller's anatomy of the dog-E-Book. Elsevier Health Sciences

Fechner GT (1860) Elemente der psychophysik, vol 2. Breitkopf u. Härtel

Fedurek P, Neumann C, Bouquet Y, Mercier S, Magris M, Quintero F, Zuberbühler K (2019) Behavioural patterns of vocal greeting production in four primate species. Royal Society open science 6(4):182181

Feigenson L, Dehaene S, Spelke E (2004) Core systems of number. Trends in cognitive sciences 8(7):307-314

Forstmeier W, Schielzeth H (2011) Cryptic multiple hypotheses testing in linear models: overestimated effect sizes and the winner's curse. Behavioral ecology and sociobiology $65(1): 47-55$

Foyer P, Wilsson E, Jensen P (2016) Levels of maternal care in dogs affect adult offspring temperament. Scientific reports $6(1): 1-8$

Franzen L, Delis I, De Sousa G, Kayser C, Philiastides MG (2020) Auditory information enhances post-sensory visual evidence during rapid multisensory decision-making. Nature communications 11(1):1-14 
Ginsburg N, Nicholls A (1988) Perceived numerosity as a function of item size. Perceptual and Motor Skills 67(2):656-658

Haehner A, Rodewald A, Gerber JC, Hummel T (2008) Correlation of olfactory function with changes in the volume of the human olfactory bulb. Archives of otolaryngology-head \& neck surgery 134(6):621-624

Hall NJ, Glenn K, Smith DW, Wynne CD (2015) Performance of pugs, german shepherds, and greyhounds (canis lupus familiaris) on an odor-discrimination task. Journal of Comparative Psychology 129(3):237

Hecht EE, Smaers JB, Dunn WD, Kent M, Preuss TM, Gutman DA (2019) Significant neuroanatomical variation among domestic dog breeds. Journal of Neuroscience 39(39):7748-7758

Hector A, Von Felten S, Schmid B (2010) Analysis of variance with unbalanced data: an update for ecology \& evolution. Journal of animal ecology 79(2):308-316

Hepper PG, Wells DL (2005) How many footsteps do dogs need to determine the direction of an odour trail? Chemical Senses 30(4):291-298

Horowitz A (2014) Domestic dog cognition and behavior. The Scientific Study of Canis familiaris

Horowitz A (2021) Considering the "dog" in dog-human interaction. Frontiers in Veterinary Science 8:299

Horowitz A, Hecht J, Dedrick A (2013) Smelling more or less: Investigating the olfactory experience of the domestic dog. Learning and motivation 44(4):207-217

Jackson SM, Martin GK, Roberts WA (2021) The olfactory capability of dogs to discriminate between different quantities of food. Learning \& Behavior pp 1-9

Jezierski T, Adamkiewicz E, Walczak M, Sobczyńska M, Gorecka-Bruzda A, Ensminger J, Papet E (2014) Efficacy of drug detection by fully-trained police dogs varies by breed, training level, type of drug and search environment. Forensic Science International $237: 112-118$ 
Kavoi BM, Jameela H (2011) Comparative morphometry of the olfactory bulb, tract and stria in the human, dog and goat

Kerepesi A, Jonsson G, Miklósi Á, Topál J, Csányi V, Magnusson M (2005) Detection of temporal patterns in dog-human interaction. Behavioural processes 70(1):69-79

Lévy F, Keller M, Poindron P (2004) Olfactory regulation of maternal behavior in mammals. Hormones and behavior 46(3):284-302

Lindsay SR (2013) Handbook of applied dog behavior and training, adaptation and learning, vol 1. John Wiley \& Sons

Lisberg AE, Snowdon CT (2009) The effects of sex, gonadectomy and status on investigation patterns of unfamiliar conspecific urine in domestic dogs, canis familiaris. Animal Behaviour 77(5):1147-1154

McGreevy P, Grassi TD, Harman AM (2004) A strong correlation exists between the distribution of retinal ganglion cells and nose length in the dog. Brain, behavior and evolution 63(1):13-22

McGreevy PD, Georgevsky D, Carrasco J, Valenzuela M, Duffy DL, Serpell JA (2013) Dog behavior co-varies with height, bodyweight and skull shape. PloS one 8(12):e80529

Mercier MR, Cappe C (2020) The interplay between multisensory integration and perceptual decision making. NeuroImage 222:116970

Pannunzi M, Nowotny T (2019) Odor stimuli: not just chemical identity. Frontiers in physiology 10:1428

Petrazzini MEM, Wynne CD (2016) What counts for dogs (canis lupus familiaris) in a quantity discrimination task? Behavioural processes 122:90-97

Petrazzini MEM, Wynne CD (2017) Quantity discrimination in canids: Dogs (canis familiaris) and wolves (canis lupus) compared. Behavioural processes 144:89-92

Petrazzini MEM, Mantese F, Prato-Previde E (2020) Food quantity discrimination in puppies (canis lupus familiaris). Animal cognition 23(4):703-710 
Pfeifer R, Iida F, Bongard J (2005) New robotics: Design principles for intelligent systems. Artificial life 11(1-2):99-120

Piazza M (2011) Neurocognitive start-up tools for symbolic number representations. Space, time and number in the brain pp 267-285

Polgár Z, Kinnunen M, Újváry D, Miklósi Á, Gácsi M (2016) A test of canine olfactory capacity: comparing various dog breeds and wolves in a natural detection task. PloS one 11(5):e0154087

Prato-Previde E, Marshall-Pescini S, Valsecchi P (2008) Is your choice my choice? the owners' effect on pet dogs'(canis lupus familiaris) performance in a food choice task. Animal Cognition 11(1):167-174

Quignon P, Kirkness E, Cadieu E, Touleimat N, Guyon R, Renier C, Hitte C, André C, Fraser C, Galibert F (2003) Comparison of the canine and human olfactory receptor gene repertoires. Genome biology 4(12):1-9

Roberts WA (2010) Distance and magnitude effects in sequential number discrimination by pigeons. Journal of Experimental Psychology: Animal Behavior Processes 36(2):206

Robin S, Tacher S, Rimbault M, Vaysse A, Dréano S, André C, Hitte C, Galibert F (2009) Genetic diversity of canine olfactory receptors. BMC genomics 10(1):1-16

Schöpper LM, Hilchey MD, Lappe M, Frings C (2020) Detection versus discrimination: The limits of binding accounts in action control. Attention, Perception, \& Psychophysics 82(4):2085-2097

Selba MC, Bryson ER, Rosenberg CL, Heng HG, DeLeon VB (2021) Selective breeding in domestic dogs: How selecting for a short face impacted canine neuroanatomy. The Anatomical Record 304(1):101-115

Soproni K, Miklósi Á, Topál J, Csányi V (2002) Dogs'(canis familaris) responsiveness to human pointing gestures. Journal of comparative psychology 116(1):27

Tacher S, Quignon P, Rimbault M, Dreano S, Andre C, Galibert F (2005) Olfactory receptor sequence polymorphism within and between breeds of dogs. Journal of Heredity 96(7):812-816 
Teng KT, McGreevy PD, Toribio JAL, Dhand NK (2016) Trends in popularity of some morphological traits of purebred dogs in australia. Canine genetics and epidemiology $3(1): 1-9$

Ward C, Smuts BB (2007) Quantity-based judgments in the domestic dog (canis lupus familiaris). Animal cognition 10(1):71-80 